\title{
Brain areas involved in the acupuncture treatment of AD model rats: a PET study
}

\author{
Yangjia Lu, ${ }^{1,4}$ Yong Huang ${ }^{1 *}$, Chunzhi Tang ${ }^{2}$, Baoci Shan ${ }^{3}$, Shaoyang Cui ${ }^{2,5}$, Junjun Yang ${ }^{2}$, Junqi Chen¹,
} Renyong Lin ${ }^{1}$, Huiling Xiao', Shanshan $\mathrm{Qu}^{1}$ and Xinsheng Lai ${ }^{2^{*}}$

\begin{abstract}
Background: Acupuncture may effectively treat certain symptoms of Alzheimer's disease (AD). Although several studies have used functional brain imaging to investigate the mechanisms of acupuncture treatment on AD, these mechanisms are still poorly understood. We therefore further explored the mechanism by which needling at ST36 may have a therapeutic effect in a rat AD model.

Methods: A total of 80 healthy Wistar rats were divided into healthy control $(n=15)$ and pre-model $(n=65)$ groups. After inducing AD-like disease, a total of $45 \mathrm{AD}$ model rats were randomly divided into three groups: the model group ( $n=15)$, the sham-point group $(n=15)$, and the ST36 group $(n=15)$. The above three groups underwent PET scanning. PET images were processed with SPM2.

Results: The brain areas that were activated in the sham-point group relative to the model group were primarily centred on the bilateral limbic system, the right frontal lobe, and the striatum, whereas the activated areas in the ST36 group were primarily centred on the bilateral limbic system (pyriform cortex), the bilateral temporal lobe (olfactory cortex), the right amygdala and the right hippocampus. Compared with the sham-point group, the ST36 group showed greater activation in the bilateral amygdalae and the left temporal lobe.
\end{abstract}

Conclusion: We concluded that needling at a sham point or ST36 can increase blood perfusion and glycol metabolism in certain brain areas, and thus may have a positive influence on the cognition of AD patients.

Keywords: PET, Needling, ST36, Rat, AD

\section{Background}

Alzheimer's disease (AD) is the most common form of dementia in elderly individuals and is associated with progressive memory loss and cognitive dysfunction. AD is characterised by extensive cortical neuropathology, including extracellular amyloid-containing neural plaques, intracellular neurofibrillary tangles, and cell and synapse loss in the cortical and subcortical regions of the human brain [1-4]. Research is being performed around the world to find a cure for this disease.

Acupuncture has therapeutic effects on Alzheimer's disease [5-10]. Many studies provide different explanations as to how acupuncture may cure Alzheimer's

\footnotetext{
*Correspondence: nanfanglihuang@163.com; lai1023@163.com 'School of Traditional Chinese Medicine, Southern Medical University, Guangzhou 510515, China

${ }^{2}$ Clinical Medical College of Acupuncture, Moxibustion and Rehabilitation, Guangzhou University of Chinese Medicine, Guangzhou, China Full list of author information is available at the end of the article
}

disease [11,12]. However, the mechanism is still unknown. This study was based on functional brain imaging using positron emission tomography (PET) to explore the mechanism of the therapeutic effect of acupuncture on $\mathrm{AD}$. PET is a non-invasive imaging technique and can reveal the level of cellular metabolism. This technique has been used to explore acupoint specificity and the relationship between needling specificity and the response in cerebral regions [13]. Jia et al. [14], Dong et al. [15], and Yin et al. [16,17] have all performed similar studies based on PET to determine how needling works. Their results showed that needling at different acupoints can stimulate different brain regions. Our literature review revealed the following: (1) All of these studies were performed on humans, and there have been no PET studies to determine the effects of needling in rats. (2) No functional brain imaging studies have been performed on rat models of AD. This study is the first 
to study the needling of rats using PET. We selected ST-36 (Zhusanli) as the needling point with the aim of determining how needling at ST-36 affects the brain.

\section{Methods}

Design

A randomised, controlled animal experiment.

\section{Time and setting}

The study was performed at the Experimental Animals Center of the China Academy of Chinese Medical Sciences, the PET-CT centre of the Experimental Animals Center of the General Hospital of PLA, and the Institute of High Energy Physics of the Chinese Academy of Sciences from January 2011 to October 2012.

\section{Ethical statement}

Animal care and sacrifice were conducted according to methods approved by the Animal Care and Use Committee, Guangzhou University of Traditional Chinese Medicine (TCM), Guangzhou, China. All experiments were performed in accordance with the National Institute of Health Guide for the Care and Use of Laboratory Animals. This experiment was approved by the Ethics Committee of Guangzhou University of TCM (SPF20110032).

\section{Materials}

\section{Reagents and instruments}

Reagents and instruments were used in this research were showed as Table 1.

\section{Experimental animals}

A total of 80 healthy 8-week-old Wistar rats (40 males and 40 females) weighing $200-250 \mathrm{~g}$ were provided by the animal centre of the China Academy of Chinese Medical Sciences. The entire experimental procedure was in accordance with the Guidelines for the Care and Use of Laboratory Animals of the Ministry of Science and Technology of the People's Republic of China.

\section{Methods}

\section{Primary experimental groups}

The 80 rats were bought from the animal laboratory of Guangzhou University of TCM, Guangzhou, and housed in the Experimental Animals Center of the China Academy of Chinese Medical Sciences. All rats were housed in separate cages under conditions of controlled illumination $(12: 12 \mathrm{~h}$ light/dark cycle), humidity, and temperature $\left(18-22^{\circ} \mathrm{C}\right)$. After 1 week of acclimatisation to the home cage, the rats were randomly assigned to the healthy control $(\mathrm{n}=15,8$ males and 7 females) or pre-model $(n=65,32$ males and 33 females) groups.

\section{Generation of the animal model}

The healthy control (HC) group was not subjected to any treatment. The pre-model group received $\mathrm{D}$-gal (20 $\mathrm{mg}$ in $2 \mathrm{ml} 0.9 \%$ saline, intraperitoneal injection every day) for 6 weeks. After 6 weeks of intraperitoneal D-gal injections, the pre-model rats were anesthetised with pentobarbital $(50 \mathrm{mg} / \mathrm{kg})$. The head of each rat was placed in a stereotaxic frame, and a needle with a syringe (Hamilton) was placed in the right NBM, $0.9 \mathrm{~mm}$ posterior and $2.8 \mathrm{~mm}$ lateral to the bregma and $6.9 \mathrm{~mm}$ vertically from the skull surface. A stereotaxic injection of $6 \mathrm{Ag}$ of ibotenic acid in $0.3 \mathrm{Al}$ of phosphate-buffered saline ( $\mathrm{pH} 7.4$ ) was performed at a rate of $0.1 \mathrm{Al} / \mathrm{min}$. The syringe was left in place for more than $3 \mathrm{~min}$ at the site of injection. The position of the lesion was confirmed using Nissl staining. Two weeks after the IBO injection [18-20], all of the animals (including the $\mathrm{HC}$ and pre-model animals) were subjected to the Y-maze test. The rats in the pre-model group that met the standards of the AD model were included in the next step. A total

Table 1 Reagents and instruments used in this study

\begin{tabular}{|c|c|c|}
\hline Reagents and instruments & Type & Source \\
\hline D-Galactose (d-gal) & - & Shanghai No. 2 reagent company \\
\hline Ibotenic acid (IBA) & - & Sigma Chemical Co. P.O. \\
\hline sodium pentobarbital & - & Beijing Chemical Reagent Company \\
\hline sodium benzylpenicillin & - & Harbin Group Pharmaceutical Factory \\
\hline Normal sodium, NS & - & Guangxi Nanning Bai-hui Pharmaceutical Group Co., LTD \\
\hline stereotaxic apparatus & WDT-2 & Xian Northwest Photoelectric Instrument Factory \\
\hline${ }^{18}$ F-FDG & - & General Hospital of PLA \\
\hline MicroPET imaging system & nanoScan PET & SIEMENS, Germany \\
\hline ECAT EXACT HR + PET imaging system & $\mathrm{HR}+$ & SIEMENS, Germany \\
\hline Animal breathing anaesthesia machine & VME & Matrx Company, America \\
\hline isoflurane & - & Hebei Jiu-pai Pharmaceutical Co., LTD \\
\hline Rat Y-maze & RD1102-YM-R & Zhenhua Teaching Instrument Yuanyang, Hebei \\
\hline
\end{tabular}


of 45 rats (23 males and 22 females) that fulfilled the criteria of the AD model (10 rats, 4 males and 6 females, died during the D-gal treatment, and 5 rats, 3 males and 2 females, did not meet the criteria for the AD model) and 15 healthy rats were included.

\section{Secondary experimental groups}

The 45 AD model rats were divided into three groups using a random number table: the model group $(\mathrm{n}=15,7$ males and 8 females), the sham-point group ( $\mathrm{n}=15,7$ males and 8 females), and the ST36 group ( $\mathrm{n}=15,8$ males and $7 \mathrm{fe}-$ males). The three groups and the $\mathrm{HC}$ group underwent PET scanning.

\section{ST36 and sham point location}

The ST36 point is located $5 \mathrm{~mm}$ directly below the capitulum fibulae, and the sham-point used was located $2 \mathrm{~mm}$ away from ST36 (Figure 1).

\section{Needling method}

Needling was performed on rats in the sham-point group and the ST36 group the day after the AD model rats were assigned to groups. Needling was performed at the Experimental Animals Center of the China Academy of Chinese Medical Sciences, from 08:00 to 12:00. Each rat was needled one time per day, and each single needling lasted for almost $30 \mathrm{~min}$. During needling, rats were fixed only by the hand of the operator; furthermore, they were penetrated bilaterally. Needling was performed as follows: after the overlying skin was sterilised with an iodine tincture and alcohol, sterile acupuncture needles $(0.10 \mathrm{~mm}$ in diameter and $0.9 \mathrm{~mm}$ in length, manufactured by the Shuzhou Medical Appliance Factory, Shuzhou, China) were inserted perpendicularly into the selected points to a depth of $0.5-0.6 \mathrm{~mm}$. The needles were gently twisted, lifted and reinserted for even reinforcing and reducing. The twisting was performed within the range of $90-180^{\circ}$ at a rate of $60-90$ times $/ \mathrm{min}$. The lifting and thrusting was performed within the range of approximately $0.1-0.2 \mathrm{~mm}$ at a rate of $60-90 \mathrm{times} / \mathrm{min}$.

\section{${ }^{18}$ F] fludeoxyglucose (FDG)-PET imaging}

All rats were sent to the PET-CT centre of the Experimental Animals Center of the General Hospital of PLA at 8:00 am after $24 \mathrm{~h}$ of fasting and were then subjected to the following procedure: (1) The blood sugar level was determined. (2) The rats were allowed to rest for 20 min in a dark room. (3) A tracer $\left({ }^{18} \mathrm{~F}\right.$-FDG, synthesised with Mini Tracer accelerator, $0.11 \mathrm{mci} / \mathrm{kg}$ dosage) was injected via the tail vein. (4) The rats were allowed to rest for $40 \mathrm{~min}$. After tracer injection, the $\mathrm{HC}$ group and the model group rats were allowed to move freely in a small box for $30 \mathrm{~min}$. Then, $30 \mathrm{~min}$ later, the $\mathrm{HC}$ group and the model group rats received gas (isoflurane) anaesthesia; the total time of gas anaesthesia was almost 10 min. The sham-point group rats and the ST36 group rats underwent needling 2 min before the $\left[{ }^{18}\right.$ F]FDG injection. $\left[{ }^{18} \mathrm{~F}\right] \mathrm{FDG}$ was injected via the tail vein with the needle still in the acupoint (or sham-point). After finishing the injection (which was performed over almost $1 \mathrm{~min})$, the manipulation of the needle was continued as before for an additional $7 \mathrm{~min}$. The total needling time was approximately $10 \mathrm{~min}$. Then, the rats were allowed to move freely in a small box, as for the model group. Twenty minutes later, the rats were placed in a gas anaesthesia box for anaesthesia, and 10 min later, when the rats were fully anaesthetised, they underwent PET scanning. (5) The rats were subjected to a PET scan. PET scans were performed on a Biograph Duo BGO scanner (Siemens, Germany). The images covered the whole brain and were parallel to the AC-PC line. Image acquisition was started after a 40 min uptake period (bed: 1; collection mode: 3D; slice thickness: $3 \mathrm{~mm}$; slice interval: $1.5 \mathrm{~mm}$; matrix size: $256 \times 256$; total counts: $3 \times 109$ ) . Upon the completion of data acquisition, the images were reconstructed using ordered subset expectation maximisation (OSEM) with 6 iterations and 16 subsets.

\section{Statistical analysis}

The preprocessing and data analysis were performed using our self-developed toolbox for voxel-wise analysis

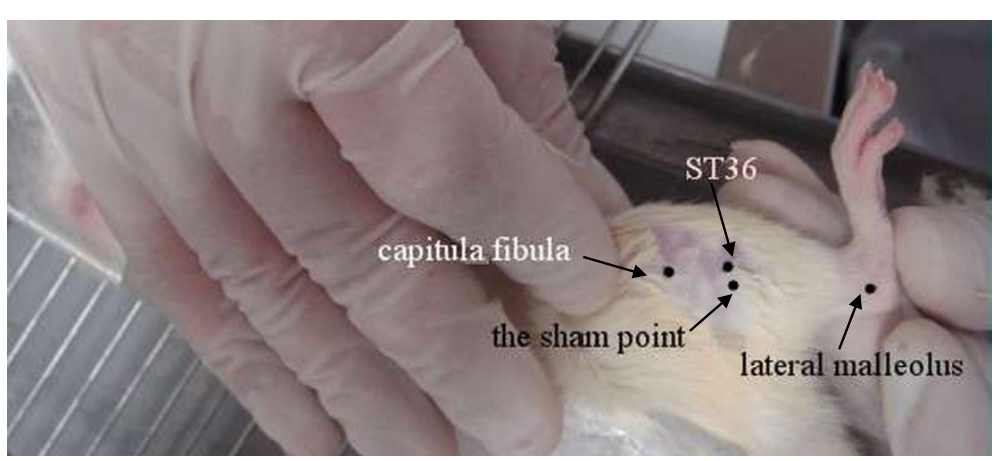

Figure 1 Location of ST36 and the sham point. 
Table 2 Sham-point group compared with the model group

\begin{tabular}{llllll}
\hline Anatomical structure & Volume & T-Average & $\mathbf{x}$ & $\mathbf{y}$ & $\mathbf{z}$ \\
\hline R Limbic System: Septal Area & 173 & 3.853346 & 41 & 16 & 62 \\
R Frontal Lobe: Orbital Cortex & 114 & 3.771888 & 44 & 36 & 78 \\
L Corpus Callosum & 137 & 3.714816 & 73 & 39 & 78 \\
L Nucleus Accumbens & 699 & 4.278525 & 71 & 26 & 73 \\
L Limbic System: Septal Area & 140 & 3.916688 & 61 & 26 & 72 \\
L Striatum & 1395 & 3.990151 & 82 & 32 & 71 \\
\hline
\end{tabular}

of rat brain images based on SPM8 (Welcome Department of Cognitive Neurology, London, UK), which comprised a FDG-PET rat brain template and atlas in Paxinos \& Watson space [21]. The images of all rats in the model group, the sham-point group, the ST36 group and the healthy controls were preprocessed using the following main steps. (1) Segmentation. All the FDGPET functional images were registered to the average rat brain with extracranial tissues using intensity-based twelve-parameter affine transformation. The intracranial mask image was employed to automatically crop brain tissues from non-brain tissues in all images. (2) Normalisation. Each intracranial individual image was spatially normalised with the intracranial FDG-PET rat brain template in SPM8. (3) Smoothing. The spatially normalised functional images were smoothed by a Gaussian kernel of $2 \% 4$ (after zooming) full width at halfmaximum (FWHM). Preprocessed images were analysed within SPM8 based on the framework of the general linear model. To identify the difference of FDG signals between the rats with $\mathrm{AD}$ and the healthy controls, two- sample t-test was performed using SPM8. Proportional scaling was applied to account for global confounds. Brain regions with significant FDG changes in rats with AD were yielded based on a voxel-level height threshold of $\mathrm{p}<0.001$ (uncorrected) and a cluster-extent threshold of 50 voxels.

\section{Results}

Compared with the model group, the sham-point group exhibited a higher level of glycol metabolism in the right nucleus accumbens, the septal area of the right limbic system, the orbital cortex of the right frontal lobe, the left corpus callosum, the left nucleus accumbens, and the left striatum (Table 2, Figure 2). Compared with the model group, the ST36 group exhibited a higher level of glycol metabolism in the pyriform cortex of the right limbic system, the olfactory cortex of the right temporal lobe, the right amygdaloid body, the right hippocampus, the pyriform cortex of the left limbic system, and the olfactory cortex of the left temporal lobe (Table 3, Figure 3). Compared with the sham-point group, the ST36 group showed a higher level of glycol metabolism in the right amygdaloid body, the olfactory cortex of the left temporal lobe, and the left amygdaloid body (Table 4, Figure 4).

\section{Discussion}

It is true that studies on acupoints should be performed in humans, but we believe that studies conducted with animals also have irreplaceable significance. To date, several studies of acupuncture using functional brain imaging have been performed. However, all we know from

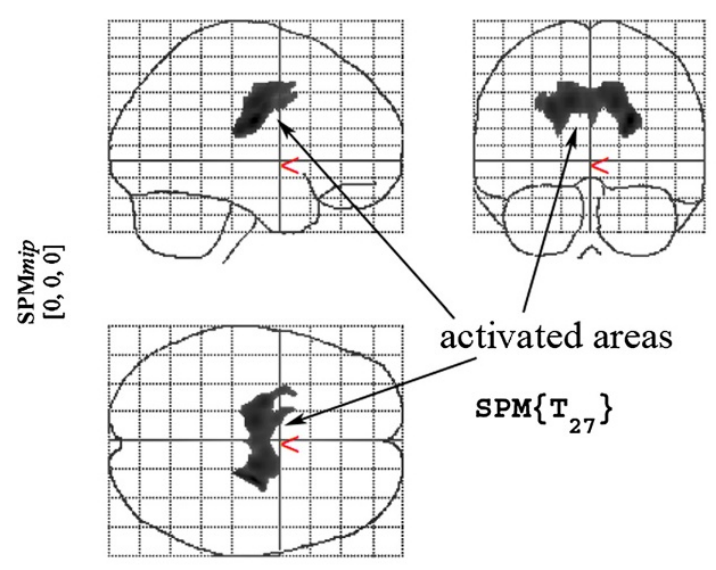

SPMresults:|result_puremodel-im_feixue

Height threshold $T=3.42$

Extent threshold $\mathbf{k}=50$ voxels

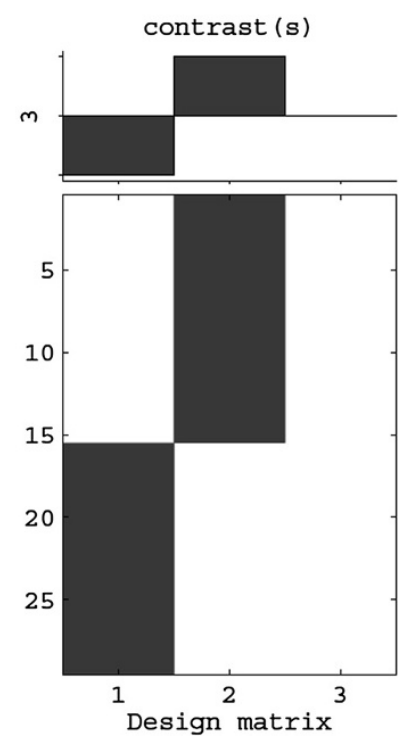

Figure 2 Cerebral areas activated in the sham-point group vs. the model group. 
Table 3 ST36 group compared with the model group

\begin{tabular}{lllccc}
\hline Anatomical structure & Volume & T-Average & $\mathbf{x}$ & $\mathbf{y}$ & $\mathbf{z}$ \\
\hline R Limbic System: Piriform Cortex & 115 & 3.799693 & 14 & 26 & 46 \\
R Temporal Lobe: Olfactory Cortex & 172 & 4.328225 & 14 & 24 & 41 \\
R Amygdaloid Body & 364 & 3.790134 & 16 & 28 & 50 \\
R Hippocampus & 119 & 4.208104 & 16 & 26 & 45 \\
L Limbic System: Piriform Cortex & 120 & 3.587087 & 107 & 25 & 49 \\
L Temporal Lobe: Olfactory Cortex & 115 & 3.606412 & 107 & 27 & 52 \\
\hline
\end{tabular}

the past studies is that needling at certain acupoints can stimulate certain brain regions. Additionally, how these activated brain regions affect the target organs and cure diseases remains unknown. Many researchers have thought about this gap in knowledge and have appealed to others to perform studies in this field $[22,23]$. However, these studies may have negative consequences on human subjects, and may require the analysis of brain tissue or another organ that cannot obtained from live humans. In contrast, it is easy to take these tissues from rats. Therefore, it may be best to perform preliminary studies in rats.

ST36 is the primary point of the four main acupoints used in traditional medical practice that has distinctive accommodation and therapeutic effects. This point is associated with more than ten common diseases, including digestive, respiratory, circulatory, urinary and nervous disorders. ST36 has been used for a long time in TCM as the main point to treat $\mathrm{AD}$. In addition, many recent studies have demonstrated that ST36 plays an important role in the treatment of $\mathrm{AD}[24,25]$. Based on these factors, we chose ST36 as the research point.
The results of this study can be grouped into three parts: (1) the brain regions with higher glycol metabolism in the sham-point group relative to the model group, (2) the brain regions with higher glycol metabolism in the ST36 group relative to the model group, and (3) the brain regions with higher glycol metabolism in the ST36 group relative to the sham-point group.

Many functional brain-imaging studies have investigated the effect of needling at ST36. Yin l et al. used PET to perform functional brain imaging while needling the ST36 points of six healthy volunteers, and they found that needling at ST36 can increase glycol metabolism in the hypothalamus, the head of the caudate nucleus, the temporal lobe, the left cerebellum, the postcentral gyrus, and the brain stem [17]. Wu Zhiyuan et al. used the BOLD sequence to observe the changes in the brain when performing acupuncture at the right ST36 of 12 healthy volunteers. The data were analysed and compared using SPM2, and this analysis revealed that there were increases in brain activity in the left parahippocampal gyrus, the left middle temporal gyrus, the left superior temporal gyrus, the right superior temporal gyrus and the right supramarginal gyrus when acupuncturing ST36 [26]. A study by Zhou investigating the effect of needling at ST36 in AD showed very similar result to our study. The differences between the two studies were as follows: (1) Zhou used fMRI, but we used PET; and (2) Zhou studied humans, but we studied rats. Zhou found that the activated regions were primarily the inferior frontal gyrus, the middle frontal gyrus, the superior frontal gyrus, the transverse temporal gyrus, the left cerebrum, the left superior frontal gyrus, the middle frontal gyrus, the precentral gyrus, the hippocampal gyrus, the

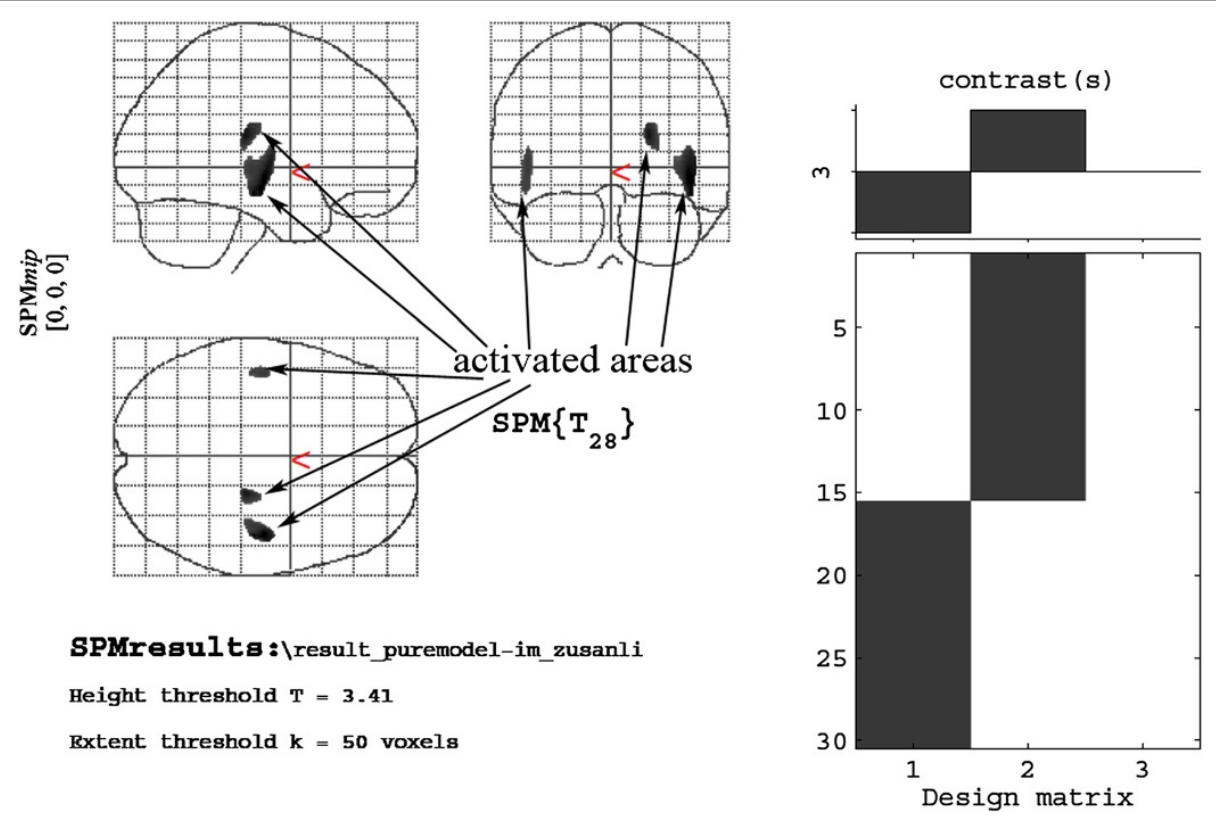

Figure 3 Cerebral areas activated in the ST36 group vs. the model group. 
Table 4 ST36 group compared with the sham-point group

\begin{tabular}{llllll}
\hline Anatomical structure & Volume & T-Average & $\mathbf{x}$ & $\mathbf{y}$ & $\mathbf{z}$ \\
\hline R Amygdaloid Body & 170 & 4.421505 & 19 & 22 & 50 \\
L Temporal Lobe: Olfactory Cortex & 117 & 4.256156 & 107 & 27 & 54 \\
L Amygdaloid Body & 128 & 4.125938 & 96 & 19 & 56 \\
\hline
\end{tabular}

cingulate gyrus, the postcentral gyrus, and the paracentral lobule of the right cerebrum. Zhou concluded that exciting ST36 could improve the cognitive ability of AD patients and that the mechanism may involve the activation of the cognition-related regions of the frontal and temporal lobes and the marginal system, as well as the cognition-related region of the cerebellum [27]. Other studies have shown a higher level of glycol metabolism in the No. 1 and 2 somesthetic areas, the No. 1 and 2 body movement areas, the premotor area, the submotor area, the inferior parietal lobe, the insula lobe, the cingulate gyrus, the prefrontal lobe, the temporal lobe, the hippocampus, the head of the caudate nucleus, the lenticular nucleus, the putamen, the nucleus accumbens, the thalamus, the hypothalamus, the paraventricular nucleus, the pons Varolii and the cerebellum [28-31]. Our results were quite different from most past results but were similar to Zhou's results, which may be because Zhou's study and our study included needling at ST36 in AD instead of healthy controls.

Senile plaques, neurofibrillary tangles and neuron loss are the three main neuropathologic changes in $\mathrm{AD}$ patients. The senile plaques and neurofibrillary tangles first occur in the medial temporal lobe (MTL), which includes the entorhinal cortex, the hippocampus, the parahippocampal gyrus and the amygdala. When $\mathrm{AD}$ progress, these three main features can spread to the neopallium, including the frontal lobe and the parietal lobe, and can eventually reach the sensory cortex [32]. In recent years, researchers have carried out many studies on the central pathological mechanism of $\mathrm{AD}$. It has been generally acknowledged that in every pathologic stage of $\mathrm{AD}$, the damaged spreads to different brain regions. These damaged brain regions have lower blood perfusion than is normal for elderly individuals. A previous study provided evidence that the deficient blood perfusion in damaged brain regions may be one of the main causes of the progressive cognitive deficit in $\mathrm{AD}$ patients [33].

Our results showed that the brain areas activated in the sham-point group relative to the model group were mainly centred on the bilateral limbic system, the right frontal lobe, and the striatum, whereas the activated areas in the ST36 group were mainly centred on the bilateral limbic system (pyriform cortex), the bilateral temporal lobe (olfactory cortex), the right amygdala and the right hippocampus. Compared with the sham-point group, the ST36 group showed activated brain regions in the bilateral amygdalae and the left temporal lobe.

The limbic system contains structures that are involved in emotion, memory and sensation. It is generally acknowledged that the limbic lobe of the limbic system is primarily located in the left hemisphere cortex. Our results revealed an apparent activation of the limbic system when needling the sham-point and ST36. Therefore, needling at these two points may improve memory and cognitive function, but these results may also be due to the rats' sensations, such as pain and fear.

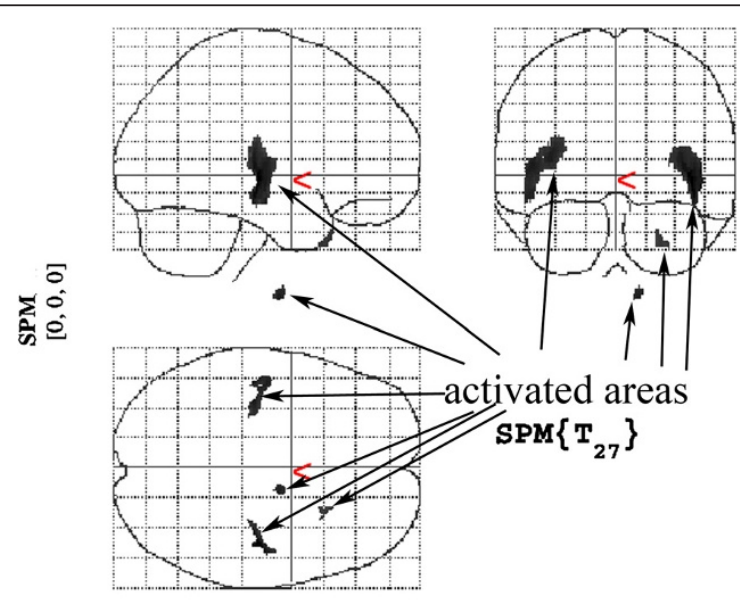

SPMresults: $\backslash$ result_im_feixue-im_zusanli

Height threshold $\mathrm{T}=3.42$

Bxtent threshold $k=50$ voxels

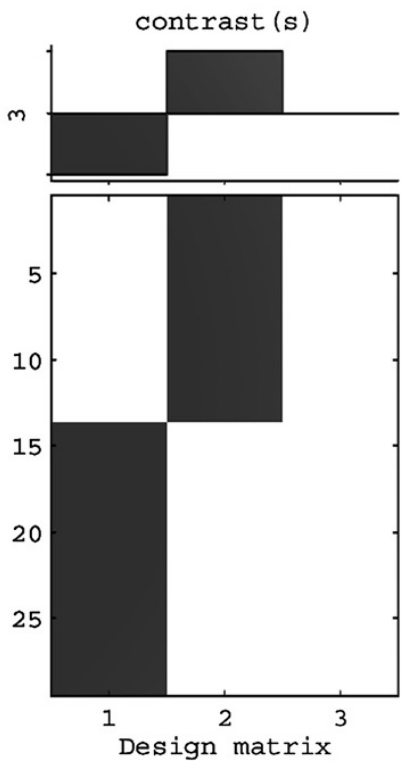

Figure 4 Cerebral areas activated by needling ST36 vs. the sham point. 
This study had several limitations: (1) Our AD model was based on the intraperitoneal injection of D-gal combined with the injection of IBO into the basal nuclei. This type of AD model rat mimics certain symptoms of $\mathrm{AD}$ but does not completely recapitulate human AD. We based our AD model on several previous studies [18-20]. (2) We tested the development of AD in rats using only a Y-maze test, and analysing some a biochemical indicator may be better.

\section{Conclusions}

According to this study, We concluded that needling at a sham point or ST36 can increase blood perfusion and glycol metabolism in certain brain areas, and thus may have a positive influence on the cognition of $\mathrm{AD}$ patients. And this may also give some helps to the clinical doctors.

\section{Competing interests}

All authors declare that they have no financial relationships with biotechnolog manufacturers, pharmaceutical companies, or other commercial entities with an interest in the subject matter or materials discussed in the manuscript.

\section{Authors' contributions}

$Y H, C Z T$ and $X L$ conceived and coordinated the study. $Y H$ and $Y L$ participated in the design of the study. $\mathrm{YL}, \mathrm{SC}$ and JC performed the study. BS performed the data analysis with SPM2. JY, RL, HX and SQ helped record the data. All authors read and approved the final manuscript.

\section{Acknowledgements}

This project was supported by the National 973 Programs of China (No. 2006CB504505, No. 2012CB518504) and the National Nature Science Foundation of China (No. 90709027).

\section{Author details}

'School of Traditional Chinese Medicine, Southern Medical University, Guangzhou 510515, China. ${ }^{2}$ Clinical Medical College of Acupuncture, Moxibustion and Rehabilitation, Guangzhou University of Chinese Medicine, Guangzhou, China. ${ }^{3}$ Key Laboratory of Nuclear Analytical Techniques, Institute of High Energy Physics, Chinese Academy of Sciences, Beijing 100049, China. ${ }^{4}$ Traditional Chinese Medicine Department of the Second Clinical School, Guangdong Medical College, Donguan, Guangdong Province, China. ${ }^{5}$ The TCM Hospital of Futian District of Shenzhen, Shenzhen, China.

Received: 5 April 2013 Accepted: 17 April 2014

Published: 31 May 2014

\section{References}

1. Cordell B: beta-Amyloid formation as a potential therapeutic target for Alzheimer's disease. Annu Rev Pharmacol Toxicol 1994, 34:69-89.

2. Marks N, Berg MJ, Saito M, Saito M: Glucosylceramide synthase decrease in frontal cortex of Alzheimer brain correlates with abnormal increSase in endogenous ceramides: consequences to morphology and viability on enzyme suppression in cultured primary neurons. Brain Res 2008 1191:136-147.

3. Akiyama H, Barger S, Barnum S, Bradt B, Bauer J, Cole GM, Cooper NR, Eikelenboom P, Emmerling M, Fiebich BL, Finch CE, Frautschy S, Griffin WS, Hampel H, Hull M, Landreth G, Lue L, Mrak R, Mackenzie IR, McGeer PL, O'Banion MK, Pachter J, Pasinetti G, Plata-Salaman C, Rogers J, Rydel R, Shen Y, Streit W, Strohmeyer R, Tooyoma I, et al: Inflammation and Alzheimer's disease. Neurobiol Aging 2000, 21(3):383-421.

4. Akiyama K, Sakurai Y, Asou H, Senshu T: Localization of peptidylarginine deiminase type II in a stage-specific immature oligodendrocyte from rat cerebral hemisphere. Neurosci Lett 1999, 274(1):53-55.
5. Liu G, Yuan LX: Clinical observation on acupuncture combined with music for treatment of Alzheimer disease. Zhongguo Zhen Jiu 2005, 25(6):390-392.

6. Peng XW, Dong KL: Clinical observation on acupuncture combined with Yizhi Jiannao granules for treatment of Alzheimer's disease. Zhongguo Zhen Jiu 2009, 29(4):269-271.

7. Zhou YL, Jia JP: Clinical observation on catgut implantation at acupoint for treatment of Alzheimer's disease. Zhongguo Zhen Jiu 2008, 28(1):37-40.

8. Zhu H, Dong KL, Wu Y, Zhang T, Li RM, Dai SS, Wang HL: Influence of acupuncture on isoprostane in patients with Alzheimer's disease. Zhongguo Zhen Jiu 2010, 30(1):18-21.

9. Guo Y, Shi X, Uchiyama H, Hasegawa A, Nakagawa Y, Tanaka M, Fukumoto I: A study on the rehabilitation of cognitive function and short-term memory in patients with Alzheimer's disease using transcutaneous electrical nerve stimulation. Front Med Biol Eng 2002, 11(4):237-247.

10. Zhou Y, Jin J: Effect of acupuncture given at the HT 7, ST 36, ST 40 and KI 3 acupoints on various parts of the brains of Alzheimer's disease patients. Acupunct Electrother Res 2008, 33(1-2):9-17.

11. Gui-mei J, Chao J, Xin-sheng L: The influence of acupuncture on neuron apoptosis in a rat model of Alzheimer Disease. Shanghai J Acupunct Moxibustion 2006, 25(12):33-36.

12. Yong-xin B, Guang-hua L: Effect of acupuncture on dysmnesia and monoamine neurotransmitter of dementia mice. Shanghai J Acupunct Moxibustion 2003, 22(7):23-25.

13. Pizzagalli DA, Oakes TR, Fox AS, Chung MK, Larson CL, Abercrombie HC, Schaefer SM, Benca RM, Davidson RJ: Functional but not structural subgenual prefrontal cortex abnormalities in melancholia. Mol Psychiatry 2004, 9(4):325-393. 405.

14. Jia SW, Wang QS, Xu WG: Study on influence of acupunctural signal on energy metabolism of human brain by positron emission tomography. Zhongguo Zhong Xi Yi Jie He Za Zhi 2002, 22(7):508-511.

15. Dong JC, Li J, Zuo CT: Influence of needling at yin-yang meridian points on cerebral glucose metabolism. Zhongguo Zhong Xi Yi Jie He Za Zhi 2002, 22(2):107-109.

16. Yin $L$, Jin $X L$, Shi $X$, Tian JH, Ma L, Yin DY, Zeng $H N$ : Imaging with pet and fmri on brain function in acupuncturing the ST-36. Chin J Rehabil Theory Prac 2002, 8(9):34-38.

17. Yin L, Jin X, Qiao W, Sun J, Shi X, Tian J, Yin D, Yao S, Shao M, Zeng H, Shan $B$, Tang Y: PET imaging of brain function while puncturing the acupoint ST36. Chin Med J (Engl) 2003, 116(12):1836-1839.

18. Hu J, Wang $\mathrm{Q}$, Liang W: Effects of bushen yizhi recipe on somatostatin-like immunopositive and somatostatin messenger ribonucleic acid expressed-positive neurons in Alzheimer's disease model rats. Zhongguo Zhong Xi Yi Jie He Za Zhi 2000, 20(7):533-535.

19. Zhong ZG, Qu ZQ, Wang NP, Zhang FF, Zhang WY, Lu UP: Effects of the Panax notoginseng saponins on the level of synaptophysin protein in brain in rat model with lesion of Meynert. Zhongguo Zhong Yao Za Zhi 2005, 30(12):913-915.

20. Zhong Z, Qu Z, Wang N, Wang J, Xie Z, Zhang F, Zhang W, Lu Z: Protective effects of Panax notoginseng saponins against pathological lesion of cholinergic neuron in rat model with Alzheimer's disease. Zhong Yao Cai 2005, 28(2):119-122.

21. Nie B, Chen K, Zhao S, Liu J, Gu X, Yao Q, Hui J, Zhang Z, Teng G, Zhao C, Shan B: A rat brain MRI template with digital stereotaxic atlas of fine anatomical delineations in paxinos space and its automated application in voxel-wise analysis. Hum Brain Mapp 2013, 34(3):1306-1318.

22. Zeng F, Liu XG, Tang Y, Liang FR: Application of PET-CT technique to the research on central mechanism of acupuncture effects. Zhen Ci Yan Jiu 2008, 33(4):284-286.

23. Jie T, Wei Q, Lin A, Peng C, Hongyan C, Baixiao Z: Preliminary study on mechanism of acupuncture in view of functional mri- - from issues to methods. J Cap Med Univ 2007, 28(6):683-687.

24. Qi O, Zhongren L, Yanyun M, Di Z, Haowen L, Qi W: Comparative study on clinical therapeutic effect of acupuncture for Alzheimer's Disease. Zhonggou Zhen Jiu 1999, 19(7):399-401.

25. Fan $Y$, Hong Z, Jing X: Effect of prescriptions for reinforcing kidney and invigorating spleen on memory of dementia rat model induced by ibotenic acid. J Nanjing Univ Tradit TCM 2008, 24(6):401-403.

26. Zhiyuan W, Fei M, Qiongyao X, Jing H, Linbao G, Genxiong N, Keming C: Comparative study on acupuncturing the different acupoints of the same meridian with functional magnetic resonance imaging. Chin $\mathrm{J}$ Med Imaging 2008, 28(8):689-693. 
27. Youlong Z, Hongyan $\mathrm{H}$, Jianping J: Correlation analysis on changes between cognitive ability and brain fmri after acupoint thread embedding in Alzheimers Disease Patients. Zhonggou Zhong Xi Yi Jie He Za Zhi 2008, 28(8):689-693.

28. Guangrui S, Ying C, Bin Y, Cheng L, GuangBin W, Qiwen T: Acupuncture of Weizhong(BL 40) and Zusanli(ST 36) on the study of brain function by PET/CT imaging. Chin J Nucl Med 2006, 26(1):54-56.

29. Xiao YY, Du L, Hong BK, Et A: Study on fMRI brain map in patients undergoing needling at Zusanli (ST36) by reinforcing method. Zhongguo Zhong Xi Yi Jie He Za Zhi 2008, 28(2):122-125.

30. Wei W, Jianpin Q, Yeling X, Xiaolin H, Wenxun L, Chengyuan W: The response of human motor cortex to acupuncture of S36 and G34 as revealed by functional MRI. Chin J Phys Med Rehabil 2004, 26(8):472-475.

31. Zhang XY, Gao S, Zhao JG, Cai L, Pang JP, Lu MX: PET study of effects of combination of different points on glucose metabolism in the patient of cerebral infarction. Zhongguo Zhen Jiu 2007, 27(1):26-30.

32. Hampel H, Teipel SJ, Alexander GE, Pogarell O, Rapoport SI, Moller HJ: In vivo imaging of region and cell type specific neocortical neurodegeneration in Alzheimer's disease. Perspectives of MRI derived corpus callosum measurement for mapping disease progression and effects of therapy. Evidence from studies with MRI, EEG and PET. J Neural Transm 2002, 109(5-6):837-855.

33. Nishimura T, Hashikawa K, Fukuyama H, Kubota T, Kitamura S, Matsuda $H$, Hanyu H, Nabatame H, Oku N, Tanabe H, Kuwanara Y, Jinnouchi S, Kubo A: Decreased cerebral blood flow and prognosis of Alzheimer's disease: a multicenter HMPAO-SPECT study. Ann Nucl Med 2007, 21(1):15-23.

doi:10.1186/1472-6882-14-178

Cite this article as: Lu et al:: Brain areas involved in the acupuncture treatment of AD model rats: a PET study. BMC Complementary and

Alternative Medicine 2014 14:178.

\section{Submit your next manuscript to BioMed Central and take full advantage of:}

- Convenient online submission

- Thorough peer review

- No space constraints or color figure charges

- Immediate publication on acceptance

- Inclusion in PubMed, CAS, Scopus and Google Scholar

- Research which is freely available for redistribution
C Biomed Central 